\title{
Impaired Degradation of Prostaglandins and Thromboxane in Zellweger Syndrome
}

\author{
JOACHIM FAULER, DIMITIRIOS TSIKAS, ERTAN MAYATEPEK, DIETRICH KEPPLER, \\ AND JÜRGEN C. FRÖLICH \\ Department of Clinical Pharmacology, Hannover Medical School, D-30625 Hannover, Germany \\ [J.F., D.T., J.C.F.]; Department of General Pediatrics, University Children's Hospital, D-69120 \\ Heidelberg, Germany [E.M.]; and Division of Tumor Biochemistry, German Cancer Research \\ Center, D-69120 Heidelberg, Germany [D.K.]
}

\begin{tabular}{|} 
ABSTR \\
Cyclooxygenase products are metabolized by $\omega$-oxida-
\end{tabular}

Cyclooxygenase products are metabolized by $\omega$-oxida-
tion as well as $\beta$-oxidation. Children with Zellweger syndrome (ZS) are characterized by peroxisome deficiency. To evaluate the role of peroxisomal $\beta$-oxidation on cyclooxygenase metabolites, the degradation of endogenous prostanglandin (PG) $E_{2}$, prostacyclin, and thromboxane (Tx) $A_{2}$ was assessed in children with $\mathrm{ZS}(n=7)$ and in healthy children $(n=7)$. $\mathrm{PGE}_{2}$, prostacyclin, $\mathrm{TxB}_{2}$, and their major urinary metabolites $7 \alpha$-hydroxy-5,11-dioxotetranor-prosta-1,16-dioic acid, 2,3-dinor-6-oxo-PGF , $_{1 \alpha}$, and 2,3-dinor- $\mathrm{TxB}_{2}$, respectively, were measured in urine by gas chromatography-mass spectrometry/mass spectrometry. The median excretion of healthy children was $17.9 \mathrm{ng}$ of $7 \alpha$-hydroxy-5,11-dioxo-tetranor-prosta-1,16dioic acid/mg creatinine (interquartile range, 6.3 to 19.4 $\mathrm{ng} / \mathrm{mg}$ ), $0.38 \mathrm{ng}$ of 2,3 -dinor-6-oxo-PGF ${ }_{1 \alpha} / \mathrm{mg}$ creatinine (interquartile range, 0.34 to $0.70 \mathrm{ng} / \mathrm{mg}$ ), and $0.36 \mathrm{ng}$ of 2,3-dinor- $\mathrm{TxB}_{2} / \mathrm{mg}$ creatinine (interquartile range, 0.14 to $0.54 \mathrm{ng} / \mathrm{mg}$ ). In contrast, none of these metabolites could be detected in urine of children with ZS $(p<0.002)$. However, we identified in the urine of these children a new metabolite of $\mathrm{PGE}_{2}$ as 11-hydroxy-9,15-dioxo-prost-5-en1,20-dioic acid by gas chromatography-mass spectrometry, and we confirmed the presence of 9,11-dihydroxy-15-oxoprost-5-en-1,20-dioic acid the main urinary metabolite of $\mathrm{PGF}_{2 \alpha}$ in ZS. Importantly, these two metabolites were only detectable in urine of children with ZS. Furthermore, we found highly elevated amounts of $\mathrm{PGE}_{2}, 6-\mathrm{oxo}-\mathrm{PGF}_{1 \alpha}$, and $\mathrm{TxB}_{2}$ in urine from children with $\mathrm{ZS}$ compared with the

$P G$ and $T \times A_{2}$ are biologically active arachidonic acid metabolites. They are involved in almost all physiologic as well as pathophysiologic conditions $(1,2)$. Extremely

Received November 30, 1993; accepted May 9, 1994.

Correspondence: Joachim Fauler, M.D., Department of Clinical Pharmacology, Hannover Medical School, Konstanty-Gutschowstr. 8, D-30 625 Hannover, Germany.

Supported by the Bundesministerium für Forschung und Technologie 01VM90020. E.M. is supported by the Deutsche Forschungsgemeinschaft. amounts eliminated by healthy children (all parameters, $p$ $<0.002$ ). The present findings demonstrate an impaired degradation of $P G$ and Tx in ZS on the level of $\beta$-oxidation. These data strongly support the hypothesis that prostanoids are exclusively $\beta$-oxidized by the peroxisomal pathway in vivo. (Pediatr Res 36: 449-455, 1994)

PG, prostaglandin
Tx, thromboxane
LT, leukotriene
PGE-MUM, prostaglandin E major urinary metabolite
(7 $\alpha$-hydroxy-5,11-dioxo-tetranor-prosta-1,16-dioic acid)
PGF-MUM, prostaglandin F major urinary metabolite
(5 $\alpha, 7 \alpha$-dihydroxy-11-oxo-tetranor-prosta-1,16-dioic acid)
RP-HPLC, reversed-phase high-performance liquid
chromatography
GC-MS, gas chromatography-mass spectrometry
GC-MS/MS, gas chromatography-mass
spectrometry/mass spectrometry
PFB, pentafluorobenzyl
CAD, collisionally activated dissociation
NICI, negative-ion chemical ionization
MO, methoxime
TMS, trimethylsilyl
ZS, Zellweger syndrome
TMSOH, trimethylsilanol
PFBOH, pentafluorobenzyl alcohol
[M-PFB] , [molecular mass-pentafluorobenzyl]

rapid catabolism in vivo restricts their action to the microenvironment of their release. The primary prostanoids are catabolized via oxidation and reduction to 15-oxo-13,14-dihydro-prostanoids $(3,4)$. These metabolites are moderately stable in plasma and are only weakly biologically active. They are degraded from $\mathrm{C} 1$ by $\beta$-oxidation to dinor and tetranor metabolites, which are further transformed via $\omega$-oxidation to $\omega$-carboxymetabolites. 
Although the initial metabolic reaction leading to 15oxo-13,14-dihydro-prostanoids is well elucidated, little is known about the site of $\beta$-oxidation of prostanoids. Experiments with isolated rat liver mitochondria have suggested that these organelles are able to $\beta$-oxidize prostanoids, and therefore it was generally accepted that mitochondrial $\beta$-oxidation is the major pathway (5). Recently, however, it was demonstrated that rat liver peroxisomes $\beta$-oxidize PG at a rate similar to or even higher than that of mitochondria (6).

Mitochondria catabolize fatty acids completely, whereas peroxisomes predominantly perform only two consecutive $\beta$-oxidations (7). This finding further supports a role of peroxisomes in vivo, because dinor as well as tetranor metabolites of eicosanoids can be detected in urine. Taken together, these results suggest that prostanoids can be $\beta$-oxidized by the peroxisomal pathway as well as by the mitochondrial pathway. It was therefore a challenge to evaluate the relative contribution of these two organelles to $\beta$-oxidation of prostanoids in vivo. Children with ZS, a rare hereditary disorder that is characterized by the complete absence of peroxisomes and in most cases by intact mitochondria $(8,9)$, are uniquely suited to investigate the contribution of peroxisomal $\beta$-oxidation of prostanoids.

Recently, we reported that $\mathrm{LT}$, which are arachidonic acid metabolites as well, are $\beta$-oxidized in peroxisomes and that $\beta$-oxidation proceeds from the $\omega$-end $(10)$. To verify our in vitro findings, we analyzed urine from children with ZS for LT metabolites (11). In contrast to healthy children, $\omega$-carboxy-tetranor-LTE $\mathrm{L}_{3}$ was not detectable in urine of children with ZS. Instead of $\mathrm{LTE}_{4}$, $\mathrm{N}$-acetyl-LTE $\mathrm{LT}_{4}$ and $\omega$-carboxy-LTE $\mathrm{L}_{4}$ accumulated in urine of these children. Furthermore, unexpectedly high amounts of $\mathrm{LTB}_{4}$ were found in urine of patients with $\mathrm{ZS}$ (11). This was surprising because in monkeys $\mathrm{LTB}_{4}$ was extensively catabolized by $\omega$-oxidation followed by $\beta$-oxidation (12). Our data, obtained from children with ZS, clearly demonstrate that LT are mainly degraded by peroxisomal $\beta$-oxidation in vivo in humans (11). However, it is difficult to compare the metabolism of LT with that of PG because LT are, for unknown reasons, exclusively metabolized starting from $\mathrm{C} 20$, whereas $\beta$-oxidation of PG commences at $\mathrm{C} 1$.

To investigate the fate by which PG are $\beta$-oxidized in humans, it is useful to measure the urinary index metabolites of endogenous PG synthesis. Studies with radiolabeled $\mathrm{PGE}_{2}, \mathrm{PGI}_{2}$, and $\mathrm{TxB}_{2}$ have shown that PGEMUM, 2,3-dinor-6-oxo- $\mathrm{PGF}_{1 \alpha}$, and 2,3-dinor- $\mathrm{TxB}_{2}$ are the major urinary metabolites of these primary cyclooxygenase products in humans (13-15). We have developed stable isotope dilution assays to identify and quantify these metabolites unequivocally by GC-MS/MS. In the present study, we measured the excretion of these metabolites and their precursors in children with ZS and in healthy children.

\section{METHODS}

Patients. Seven children with ZS and seven age- and sex-matched healthy infants were included in this study. All children exhibited the characteristic clinical and biochemical abnormalities described for ZS (8). The biochemical characteristics of the children with ZS (children designated as 2 to 8 ) have recently been published (11). Mitochondrial $\beta$-oxidation activity was found to be in the range of normal subjects (11). Convulsions were reported from all patients. None of the children with ZS had signs of cholestasis, liver failure, or impaired renal function. Urine was obtained from spontaneous micturition and stored at $-80^{\circ} \mathrm{C}$ until analysis.

Materials. The unlabeled and tetradeuterated $([3,3,4,4-$ ${ }^{2} \mathrm{H}_{4}$ ]) PG standards including PGE-MUM were a kind gift from Dr. U. Axen, The Upjohn Company (Kalamazoo, MI). PFB bromide was obtained from Aldrich (Steinheim, Germany). All tritiated compounds except for $\left[{ }^{3} \mathrm{H}\right]$ PGE-MUM were purchased from Amersham Buchler (Braunschweig, Germany). $\left[5,6-{ }^{3} \mathrm{H}_{2}\right] \mathrm{PGE}_{1}$ (sp act $53.7 \mathrm{Ci} / \mathrm{mmol}$ ) was purchased from Du Pont de Nemours (Dreieich, Germany). $\left[{ }^{3} \mathrm{H}\right]$ PGE-MUM was prepared by intraperitoneal injection of $100 \mathrm{mCi}$ of $[5,6-$ ${ }^{3} \mathrm{H}_{2}$ ]PGE ${ }_{1}$ in a 500-g male Sprague-Dawley rat. Two $\mathrm{h}$ before the application of $\left[5,6-{ }^{3} \mathrm{H}_{2}\right] \mathrm{PGE}_{1}$, the rat was treated with $3 \mathrm{mg}$ of diclofenac \{sodium[2-(2,6-dichlorophenylamino)phenylacetate]\} intraperitoneally and with $5 \mathrm{mg}$ of diclofenac s.c. to inhibit endogenous cyclooxygenase synthesis (diclofenac was a kind gift from Ciba-Geigy, Basel, Switzerland). $\left[{ }^{3} \mathrm{H}\right]$ PGE-MUM was isolated from urine using solid-phase extraction followed by RP-HPLC as described below. $\left[1,16-{ }^{18} \mathrm{O}_{2}\right]$ PGE-MUM was prepared enzymatically by incubation of the dimethylester of unlabeled PGE-MUM with pig liver esterase in $\mathrm{H}_{2}{ }^{18} \mathrm{O}\left(97.8\right.$ atom $\%{ }^{18} \mathrm{O}$, MSD Isotopes, Merck Frosst, Montreal, Canada). GC-MS/MS analysis gave $80.3 \%$ $\left[1,16^{-18} \mathrm{O}_{2}\right]$ PGE-MUM, and only $0.7 \%$ remained unlabeled.

Methoxyamine hydrochloride and N,O-bis(TMS)trifluoroacetamide were purchased from Pierce (Rockford, IL). $\left[{ }^{2} \mathrm{H}_{3}\right]$ methoxyamine hydrochloride $\left(99.8\right.$ atom\% $\left.{ }^{2} \mathrm{H}\right)$ was purchased from MSD Isotopes, Merck Frosst (Montreal, Canada). N,N-diisopropylethylamine was obtained from Sigma (Munich, Germany). Acetonitrile of gradient grade and all other chemicals were from Merck (Darmstadt, Germany). Octadecyl (C18) silica cartridges (500 $\mathrm{mg}$ ) were purchased from J. T. Baker (Deventer, The Netherlands).

Extraction of $P G$ and $T x$ from urine samples and derivatization. Urine samples $(1 \mathrm{~mL}$ from patients and $4 \mathrm{~mL}$ from controls) were spiked with a mixture containing $5 \mathrm{ng}$ each of tetradeuterated internal standard of 6-oxo$\mathrm{PGF}_{1 \alpha}, 2,3$-dinor-6-oxo-PGF ${ }_{1 \alpha}, \mathrm{TxB}_{2}$, and 2,3-dinor$\mathrm{TxB}_{2} ; 6.5 \mathrm{ng}$ of tetradeuterated $\mathrm{PGE}_{2}$; and $8 \mathrm{ng}$ of $[1,16-$ ${ }^{18} \mathrm{O}_{2}$ ]PGE-MUM, as well as tritiated standards of each of the PG. The urine was acidified to $\mathrm{pH} 3.0$ by the addition of $5 \mathrm{~N} \mathrm{HCOOH}$ and allowed to stay on ice for $30 \mathrm{~min}$. The 
samples were then applied to solid-phase extraction on C18 cartridges preconditioned with $10 \mathrm{~mL}$ of methanol and $3 \mathrm{~mL}$ of $0.05 \mathrm{~N} \mathrm{HCOOH}$. After washing with $20 \mathrm{~mL}$ of water and $2.5 \mathrm{~mL}$ of hexane and drying, the compounds were eluted with $2 \mathrm{~mL}$ of ethylacetate. The solvent was completely evaporated under nitrogen, and the residue was treated for $1 \mathrm{~h}$ at room temperature with 40 $\mu \mathrm{L}$ of pyridine/water/triethylamine, $10 / 10 / 10$ (vol/vol/ $\mathrm{vol})$. After evaporation of the solvents under nitrogen, the residue was redissolved in $10 \mu \mathrm{L}$ of methanol and diluted by addition of $100 \mu \mathrm{L}$ of acetonitrile. The PFB ester of the compounds were prepared by addition of 10 $\mu \mathbf{L}$ of $\mathrm{N}, \mathrm{N}$-diisopropylethylamine and $10 \mu \mathrm{L}$ of PFB bromide solution in acetonitrile (30 wt \%) and heating at $30^{\circ} \mathrm{C}$ for $1 \mathrm{~h}$. Again, reagents and solvents were evaporated under nitrogen and the residue was treated with 100 $\mu \mathrm{L}$ of a $2-\mathrm{g} \%$ methoxyamine hydrochloride solution in pyridine. Methoximation was performed by heating at $60^{\circ} \mathrm{C}$ for $1 \mathrm{~h}$. Pyridine was evaporated under nitrogen, and the residue was reconstituted in $90 \mu \mathrm{L}$ of methanol and stored at $-20^{\circ} \mathrm{C}$ until RP-HPLC analysis. The silylation procedure is described below.

$\boldsymbol{R P}$-HPLC. Separation of prostanoids as PFB derivatives by RP-HPLC has been shown to improve their analysis by GC-MS/MS (16). Before quantitation by GCMS/MS, we performed RP-HPLC analyses of the PFB-MO derivatives on a solvent delivery system (model 2150, LKB, Bromma, Sweden) coupled with a fraction collector. The stationary phase consisted of a column $(250 \times 4.6-\mathrm{mm}$ inner diameter) of $\mathrm{C} 18$ with $5-\mu \mathrm{m}$ particle size (Shandon, Runcom, UK). The mobile phase consisted of acetonitrile/water, $70 / 30 \mathrm{vol} / \mathrm{vol}$, and was pumped at a flow rate of $2.0 \mathrm{~mL} / \mathrm{min}$. The retention volumes of the PFB-MO derivatives of unlabeled and labeled PG were $6 \mathrm{~mL}$ for 2,3-dinor-6-oxo-PGF ${ }_{1 \alpha}$ and 2,3-dinor- $\mathrm{TxB}_{2}, 8 \mathrm{~mL}$ for 6-oxo-PGF ${ }_{1 \alpha}$ and $\mathrm{TxB}_{2}, 12 \mathrm{~mL}$ for $\mathrm{PGE}_{2}$, and $16 \mathrm{~mL}$ for PGE-MUM. Two-mL fractions were collected, the radioactivity was determined, and then the fractions were vortex-mixed with $6 \mathrm{~mL}$ of ethylacetate for $2 \mathrm{~min}$. The final recovery for all PG was in the range of $40-50 \%$. The coefficients of variation for the retention volume were lower than $5 \%$ for all PG. The phases were separated, and the organic layer was decanted and dried over sodium sulfate. The solvents were evaporated to dryness under nitrogen, and the residue was treated with $20 \mu \mathrm{L}$ of N,O-bis(TMS)trifluoroacetamide and allowed to react at $60^{\circ} \mathrm{C}$ for $1 \mathrm{~h}$.

$\boldsymbol{G C}$-MS/MS. GC-MS/MS has been shown to be the method of choice for the analysis of eicosanoids in biologic samples (17). In the present study, we used GCMS/MS to quantitate prostanoids in urine. GC-MS/MS was performed on a Finnigan MAT TSQ 45 triple-stage quadrupole mass spectrometer equipped with a Finnigan gas chromatograph, model 9611 (San Jose, CA). A fused silica capillary column OV-1 $(25-\mathrm{m} \times 0.25-\mathrm{mm}$ inner diameter, $0.25-\mu \mathrm{m}$ film thickness) from Macherey-Nagel (Düren, Germany) was used. Helium was used as a carrier gas at a pressure of $55 \mathrm{kPa}$. Injector, interface, and ion source were kept at $280^{\circ} \mathrm{C}, 290^{\circ} \mathrm{C}$, and $140^{\circ} \mathrm{C}$, respectively. For NICI, methane was used as a reagent gas at a pressure of $65 \mathrm{~Pa}$. Argon was used for CAD at a pressure of $0.2 \mathrm{~Pa}$. The collision energy was set to $14 \mathrm{eV}$ for all PG. The electron energy was $90 \mathrm{eV}$ and the emission current $200 \mu \mathrm{A}$. The electron multiplier voltage was $2500 \mathrm{~V}$. The following pairs of mass to charge ratios $(\mathrm{m} / \mathrm{z})$ for the parent ions $\left([\mathrm{M}-\mathrm{PFB}]^{-}\right)$and the corresponding daughter ions of labeled and unlabeled compounds were used: $590 / 586$ and $244 / 240$ for both 2,3-dinor-6-oxo$\mathrm{PGF}_{1 \alpha}$ and 2,3-dinor- $\mathrm{TxB}_{2} ; 618 / 614$ and $272 / 268$ for both 6-oxo-PGF $1 \alpha$ and $\mathrm{TxB}_{2} ; 528 / 524$ and $272 / 268$ for $\mathrm{PGE}_{2}$; and $641 / 637$ and 353/349 for PGE-MUM. One to $2.5 \mu \mathrm{L}$ were injected into the GC-MS/MS system in the splitless mode using oven temperature programs. The inter- and intraassay coefficients of variation for all prostanoids were within the range of 1.5 to $3.5 \%$ and 0.8 to $1.9 \%$, respectively. The detection limit of the method was about $10 \mathrm{ng} / \mathrm{L}$ for PGE-MUM and $5 \mathrm{ng} / \mathrm{L}$ for the other prostanoids.

Statistics. Statistical analysis was performed by using the Wilcoxon signed-rank test.

\section{RESULTS}

Excretion of the major urinary cyclooxygenase end metabolites in urine. The urinary excretion of PGE-MUM, 2,3dinor-6-oxo-PGF ${ }_{1 \alpha}$, and 2,3-dinor- $\mathrm{TxB}_{2}$ of children with $\mathrm{ZS}$ as well as of matched healthy children is shown in Figure 1. In children with ZS, none of these metabolites could be detected in urine despite detection limits of 10 , 5 , and $5 \mathrm{ng} / \mathrm{L}$ urine for PGE-MUM, 2,3-dinor-6-oxo$\mathrm{PGF}_{1 \alpha}$, and 2,3-dinor-TxB $\mathrm{B}_{2}$, respectively (Fig. 2). Healthy children excreted median 17.9 (interquartile range, 6.35 to 19.43) $\mathrm{ng}$ of PGE-MUM/mg of creatinine, median 0.38 (interquartile range, 0.34 to 0.70 ) $\mathrm{ng}$ of 2,3 -

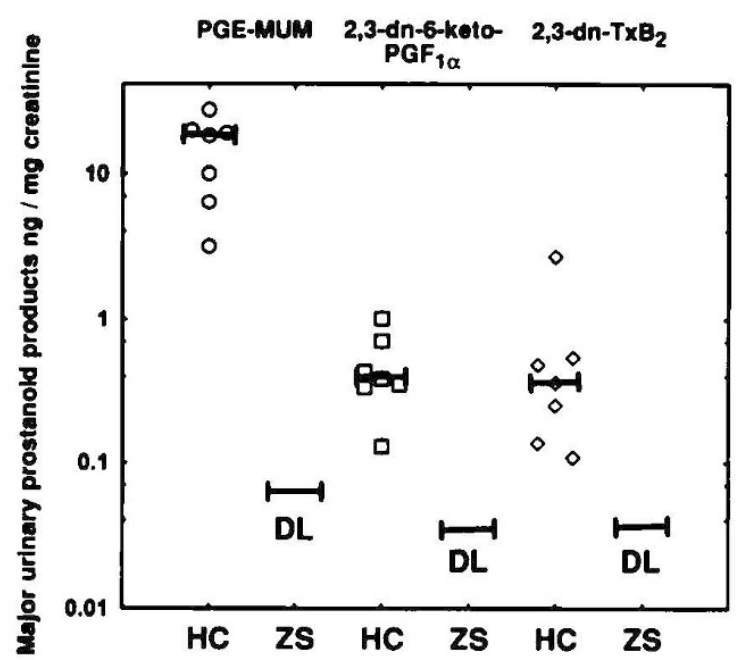

Figure 1. Urinary excretion of PGE-MUM, 2,3-dn-6-oxo-PGF ${ }_{1 \alpha}$, and 2,3-dn- $\mathrm{TxB}_{2}(\mathrm{dn}=$ dinor $)$ by healthy children $(H C)$ and by children with ZS $(Z S)$. The urinary index metabolites were measured by GC-MS/MS. Healthy children excreted significantly (all parameters, $p<0.002$ ) higher amounts of PGE-MUM, 2,3-dn-6-oxo-PGF ${ }_{1 \alpha}$, and 2,3-dn- $\mathrm{TxB}_{2}$ into urine. $D L$, Detection limit. 


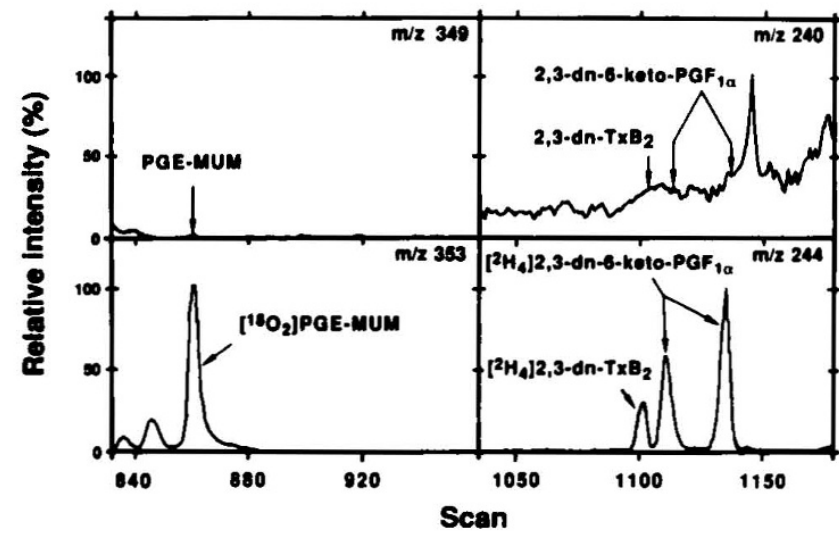

Figure 2. Partial GC-MS/MS chromatograms from the analysis of a $1-\mathrm{mL}$ aliquot from a urine sample from a child with $\mathrm{ZS}$. To this urine sample, $5 \mathrm{ng}$ each of the tetradeuterated standards of 2,3-dinor-6-oxo-

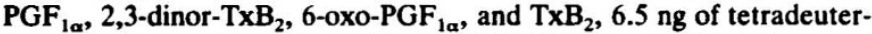
ated $\mathrm{PGE}_{2}$, and $8 \mathrm{ng}$ of $\left[1,16^{-18} \mathrm{O}_{2}\right] \mathrm{PGE}-\mathrm{MUM}$ were added. The upper panel shows tracings of endogenous urinary $P G$ in a urine sample from a child with ZS. The lower panel shows tracings of the corresponding internal standards.

dinor-6-oxo-PGF $1 \alpha / \mathrm{mg}$ of creatinine, and median 0.37 (interquartile range, 0.14 to 0.54 ) $\mathrm{ng}$ of 2,3 -dinor- $\mathrm{TxB}_{2} / \mathrm{mg}$ of creatinine into urine. The amounts of PGE-MUM excreted by healthy children were at least 180 -fold, those of 2,3-dinor-6-oxo-PGF ${ }_{1 \alpha}$ 13-fold, and those of 2,3-dinor$\mathrm{TxB}_{2}$ 10-fold higher than those excreted by children with ZS.

Excretion of primary cyclooxygenase metabolites in urine. The excretion of $\mathrm{PGE}_{2}, 6-0 x 0-\mathrm{PGF}_{1 \alpha}$, and $\mathrm{TxB}_{2}$ in urine of healthy children and children with ZS is shown in Figure 3. The children with ZS excreted significantly $(p$ $<0.002$ ) larger amounts of $\mathrm{PGE}_{2}, 6-\mathrm{oxo}_{\mathrm{PGF}}$, and $\mathrm{TxB}_{2}$ into urine compared with the healthy children. The amounts of $\mathrm{PGE}_{2}$ were 60 -fold, those of 6 -oxo-PGF 1 a

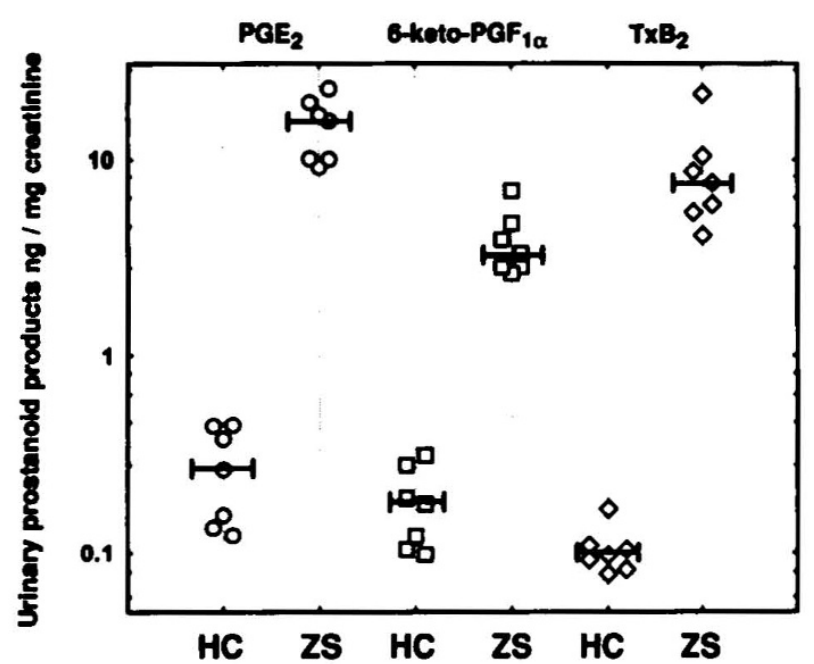

Figure 3. Urinary excretion of $\mathrm{PGE}_{2}$, 6-oxo-PGF $\mathrm{PF}_{1 \alpha}$, and $\mathrm{TxB}_{2}$ by healthy children $(H C)$ and children with ZS $(Z S)$. The primary PG were measured by GC-MS/MS. Children with ZS excreted significantly $(p<$ 0.002 ) higher amounts of $\mathrm{PGE}_{2}, 6-0 \times 0-\mathrm{PGF}_{1 \alpha}$, and $\mathrm{TxB}_{2}$ into urine than matched healthy children. 30-fold, and those of $\mathrm{TxB}_{2} 80$-fold higher than those excreted by healthy children.

Evaluation of the major urinary metabolite of the $E P G$ in ZS. A 1-mL aliquot of a urine sample from children with ZS was subjected to solid-phase extraction and the residue derivatized by PFB esterification, methoximation, and silylation as described in Methods. One- $\mu \mathrm{L}$ aliquots were injected into the GC-MS/MS system and analyzed in the NICI mode by GC-MS as well as GC-MS/MS. Figure 4 shows NICI mass spectra of the diPFB-diMO-TMS derivative of PGE-MUM (lower panel) and of the corresponding derivative of a compound with a relative retention time of 1.178 with respect to the PGE-MUM derivative (upper panel). The most intense signal in the mass spectrum of this compound was $\mathrm{m} / \mathrm{z} 691\left([\mathrm{M}-\mathrm{PFB}]^{-}\right)$, which was increased by $54 \mathrm{D}$, e.g. $\left[-\mathrm{CH}=\mathrm{CH}-\left(\mathrm{CH}_{2}\right)_{2}-\right]$, with respect to the corresponding signal of the PGE-MUM derivative at $\mathrm{m} / \mathrm{z}$ 637. Less intense signals were also found at $\mathrm{m} / \mathrm{z} 601$ and $\mathrm{m} / \mathrm{z} 511$. These were also increased by $54 \mathrm{D}$ with respect to PGE-MUM and probably resulted from consecutive fragmentation of one TMSOH group and two MO groups. This compound has two oxo groups because methoximation using ${ }^{2} \mathrm{H}_{3}$-methoxyamine hydrochloride instead of the unlabeled reagent resulted in the most intense signal at $\mathrm{m} / \mathrm{z} 697$ in the mass spectrum (data not shown), which was increased by $6 \mathrm{D}$ with respect to the unlabeled methoximated derivative (Fig. 4). Furthermore, the appearance of a less intense signal at $\mathrm{m} / \mathrm{z} 511$ but not at $\mathrm{m} / \mathrm{z} 517$ in the

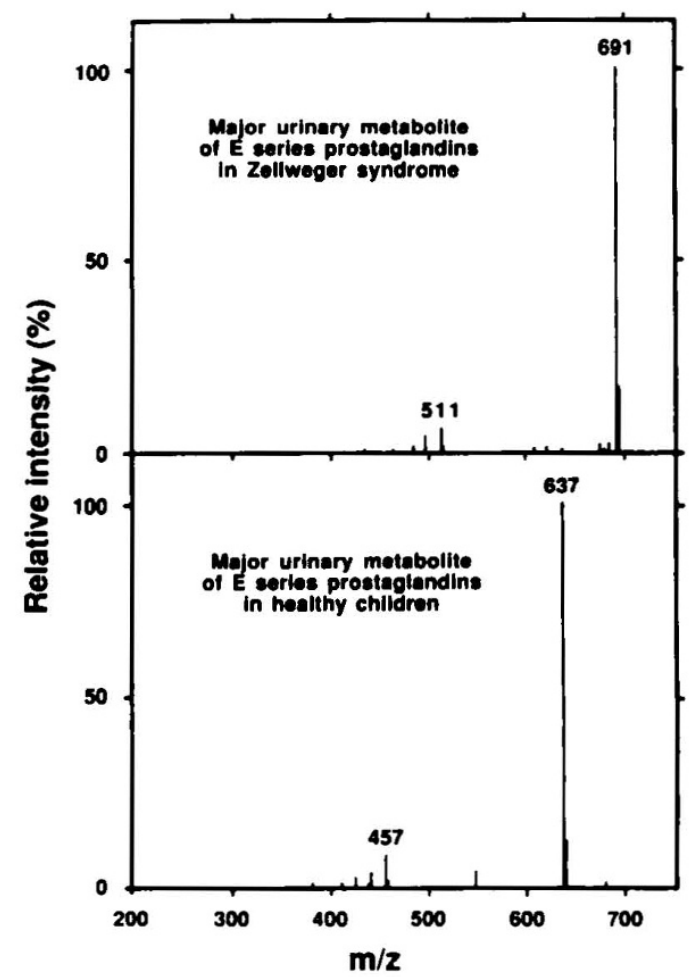

Figure 4. NICI mass spectra of the PFB-MO-TMS derivatives of synthetic PGE-MUM (lower panel) and of a metabolite extracted from the urine of a patient with ZS (upper panel). The PFB-MO-TMS derivatives of this metabolite had a relative retention time of 1.178 with respect to PGE-MUM. 
NICI mass spectrum of the ${ }^{2} \mathrm{H}_{3}$-methoximated compound demonstrates that this ion results from the release of two ${ }^{2} \mathrm{H}_{3}$-methoxy groups (96 D) and not from the release of a TMSOH group $(90 \mathrm{D})$. In addition, the PFB-MO-TMS derivative of this compound elutes from the gas chromatography column as a double peak very similar to the PFB-MO-TMS derivative of PGE-MUM. The ion at $\mathrm{m} / \mathrm{z}$ 697.7 of the PFB- $\left[{ }^{2} \mathrm{H}_{3}\right]$ MO-TMS derivative of this compound and of the corresponding ion of the PGE-MUM derivative, i.e. $\mathrm{m} / \mathrm{z} 637.7$, were subjected to $\mathrm{CAD}$ under identical conditions. CAD of the parent ion at $\mathrm{m} / \mathrm{z} 697.7$ resulted in a mass spectrum very similar to that of $\mathrm{m} / \mathrm{z}$ 637.7 of the PGE-MUM derivative (Fig. 5). All intense signals appearing in the mass spectrum of the PGE-MUM are also present in the mass spectrum of the unknown metabolite, but they all are increased by $60 \mathrm{D}$. The presence of the daughter ion at $\mathrm{m} / \mathrm{z} 499$ ([M-PFB-PFBOH $]^{-}$) in the mass spectrum of the unknown metabolite is strong evidence that this metabolite is a dicarboxylic compound. Thus, this compound is a dicarboxylic-dioxo-monoxydroxy eicosanoid. This metabolite could be exclusively identified in urine from all children with ZS by GC-MS and GC-MS/MS. Because no synthetic standard of this compound is available, exact quantitation of this new metabolite of $\mathrm{PGE}_{2}$ was not possible. However, the spectrometric

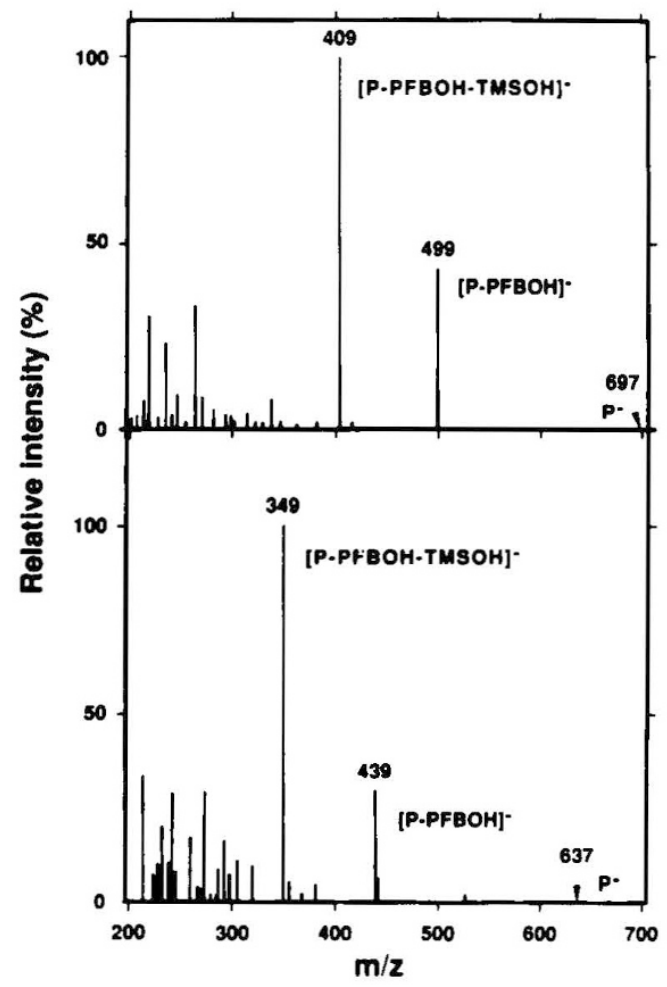

Figure 5. NICI daughter mass spectra obtained by $\mathrm{CAD}$ of the parent ions $\left([\mathrm{M}-\mathrm{PFB}]^{-}\right)\left(P^{-}\right)$of the PFB-MO-TMS derivative of synthetic PGE-MUM at $\mathrm{m} / \mathrm{z} 637.7$ and of the PFB- $\left[{ }^{2} \mathrm{H}_{3}\right]$ MO-TMS derivative at $\mathrm{m} / \mathbf{z} 697.7$ of the metabolite extracted from the urine of a patient with ZS (upper panel). The PFB-MO-TMS derivatives of this metabolite had a relative retention time of 1.178 with respect to PGE-MUM. This spectrum suggests that this new metabolite is identical with $11 \alpha$-hydroxy9,15-dioxo-prost-5-en-1,20-dioic acid, the major urinary metabolite of $\mathrm{PGE}_{2}$ in $\mathrm{ZS}$. and chromatographic data combined with the lack of PGEMUM in the urine of patients with ZS and the presence of PGE-MUM in the urine of healthy children strongly suggest that this metabolite is identical with $11 \alpha$-hydroxy-9,15dioxo-prost-5-en-1,20-dioic acid.

Major urinary metabolite of $P G F_{2 \alpha}$ in children with $\mathrm{ZS}$. In the present study, we also identified by GC-MS and GC-MS/MS (Fig. 6) the new urinary metabolite of $\mathrm{PGF}_{2 \alpha}$, which was recently discovered in urine of children with ZS (18). In the NICI mass spectrum of this metabolite, the most intense signal was observed at $\mathrm{m} / \mathrm{z} 736$ ([M$\left.\mathrm{PFB}]^{-}\right)$. CAD of this ion resulted in the formation of intense daughter ions at $\mathrm{m} / \mathrm{z} 538$ ([M-PFB-PFBOH $]^{-}$), which is characteristic for PFB esters of dicarboxylic acids; at $\mathrm{m} / \mathrm{z} 448$ ([M-PFB-PFBOH-TMSOH] $\left.]^{-}\right)$; and at $\mathrm{m} / \mathrm{z} 358$ ([M-PFB-PFBOH- $2 \times \mathrm{TMSOH}^{-}$). All these signals are increased by $54 \mathrm{D}\left[\mathrm{CH}=\mathrm{CH}-\left(\mathrm{CH}_{2}\right)_{2}\right]$ with respect to the signals of the PFB-MO-TMS derivative of PGF-MUM. On the basis of the present data, we suggest that this metabolite is identical with 9,11-dihydroxy-15oxo-prost-5-ene-1,20-dioic acid, which is identical with that described by Diczfalusy et al. (18).

\section{DISCUSSION}

To investigate whether PG and Tx are $\beta$-oxidized by the peroxisomal pathway in vivo, the major urinary metabolites of the E PG (PGE-MUM), $\mathrm{PGI}_{2}$ (2,3-dinor-6oxo-PGF ${ }_{1} \alpha$ ), and $\mathrm{TxA}_{2}$ (2,3-dinor-TxB $\mathrm{T}_{2}$ ) were assessed in urine of children with ZS and healthy children. In contrast to healthy children, none of these $\beta$-oxidized major urinary metabolites could be detected by GC-MS/MS in urine of children with ZS. The complete absence of PGF-MUM and 2,3-dinor- $\mathrm{TxB}_{2}$ in the urine from children with ZS is in line with other reports $(18,19)$. Importantly, we found a new metabolite of the E PG in urine. This new metabolite was identified by GC-MS and GC-MS/MS as 11-hydroxy-9,15-dioxo-prost-5-en-1,20-dioic acid. A hy-

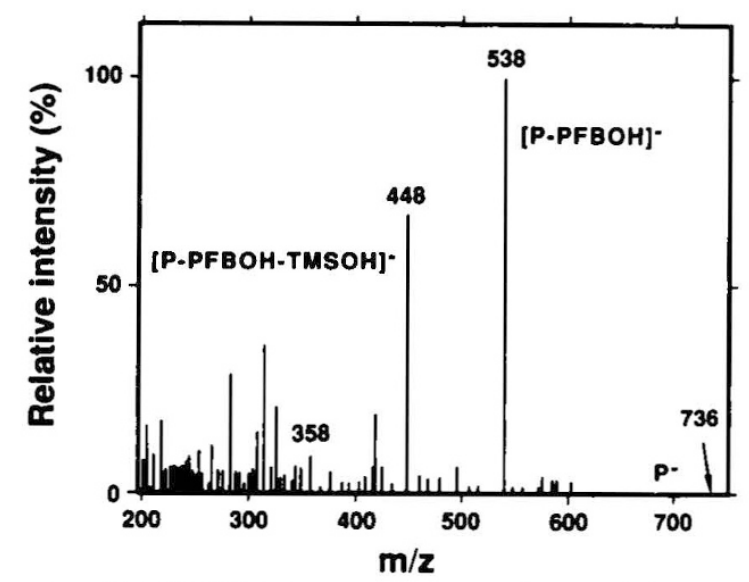

Figure 6. NICI GC-MS/MS mass spectrum of a metabolite extracted from the urine of a child with ZS the PFB-MO-TMS derivative of which had a relative retention time to the PGE-MUM derivative of 1.07. CAD of the parent ion [M-PFB] $]^{-}\left(P^{-}\right)$at $\mathrm{m} / \mathrm{z} 736$. This spectrum suggests that this metabolite is identical with $9,11 \alpha$-dihydroxy-15-oxo-prost-5-en1,20-dioic acid, the major urinary metabolite of $\mathrm{PGF}_{2 \alpha}$ in ZS. 

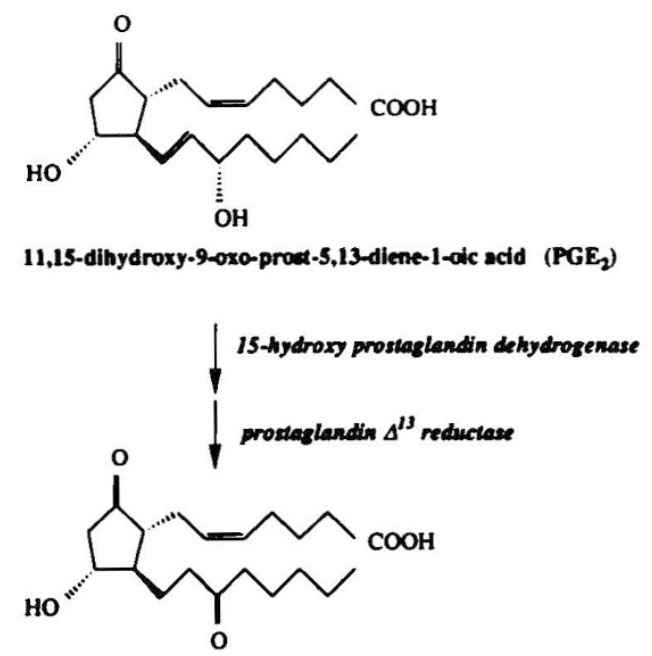

11-bydroxy-9,15-dioxa-proat-5-ene-1-oic acid (13,14-dihydro-15-0xa-PGE)

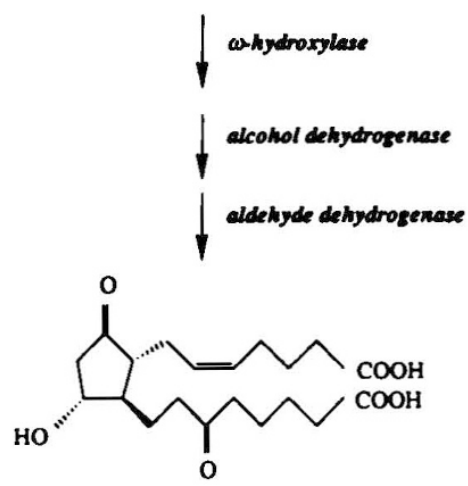

11-hydroxy-9,15-dioxo-prost-5-ene-1,20-dioic acid

Figure 7. Proposed metabolic pathway of $\mathrm{PGE}_{2}$ to $11 \alpha$-hydroxy-9,15dioxo-prost-5-en-1,20-dioic acid, the major urinary metabolite of E PG in children with ZS.

pothetical pathway for the formation of this new metabolite is shown in Figure 7. Characteristically, this new metabolite is $\omega$ - but not $\beta$-oxidized. This demonstrates that also the $E$ prostanoids are exclusively $\beta$-oxidized in peroxisomes. In addition, using the same methodology, we identified a second metabolite as 9,11-dihydroxy-15oxo-prost-5-en-1,20-dioic acid in urine from children with ZS. This metabolite is probably identical with a new major metabolite of $\mathrm{PGF}_{2 \alpha}$ in urine of children with ZS described by Diczfalusy et al. (18). Because no suitable internal standard is available, quantification of these novel metabolites by GC-MS/MS was not possible.

The fact that these two novel metabolites are only detectable in urine of children with ZS, together with the complete absence of PGE-MUM, 2,3-dinor-6-oxo$\mathrm{PGF}_{1 \alpha}$, and 2,3-dinor-TxB ${ }_{2}$, demonstrates two interesting facts. First, it reflects an impaired $\beta$-oxidation of $P G$ in these children, because the two novel metabolites are the immediate precursors of the $\beta$-oxidized PGE-MUM and PGF-MUM. Second, it shows that $\beta$-oxidation is not an essential requirement for $\omega$-oxidation of PG. In addition, $\mathrm{TxB}_{2}$ is exclusively $\beta$-oxidized in peroxisomes because 2,3-dinor- $\mathrm{TxB}_{2}$ was not detectable in urine of children with ZS. The absence of detectable amounts of urinary PGE-MUM, 2,3-dinor-6-oxo-PGF ${ }_{1 \alpha}$, and 2,3-dinor- $\mathrm{TxB}_{2}$ is apparently useful for diagnosis of impaired peroxisomal $\beta$-oxidation.

The markedly increased amounts of $\mathrm{PGE}_{2}$, 6-oxo$\mathrm{PGF}_{1 \alpha}$, and $\mathrm{TxB}_{2}$ in urine of children with $\mathrm{ZS}$ compared with the amounts excreted by healthy children is of considerable interest, because these metabolites have been shown to reflect renal PG and Tx synthesis. However, this assumption is valid only in healthy humans (20). In children with ZS, the metabolism of circulating as well as locally produced cyclooxygenase products is impaired, and therefore systemic cyclooxygenase products could be eliminated by the kidney at a much higher rate than in healthy individuals. Although this explanation is probably valid for 6-oxo-PGF ${ }_{1 \alpha}$ and $\mathrm{TxB}_{2}$, it is does not explain the enhanced excretion of $\mathrm{PGE}_{2}$ into urine because $\mathrm{PGE}_{2}$ is extremely rapidly metabolized (13) in the lung by 15 -hydroxy-dehydrogenase and ${ }^{13} \Delta$-reductase to 15 -oxo- ${ }^{13} \Delta$-dihydro-PGE 2 (4). Therefore, enhanced urinary amounts of $\mathrm{PGE}_{2}$ are most likely of renal origin (21).

On the basis of the present data, it is not possible to estimate whole-body cyclooxygenase production in children with ZS. To obtain a rough estimate of the $E$ prostanoid production in these patients, we estimated by GC-MS/MS the amounts of 9,11-dihydroxy-15-oxo-prost5-en-1,20-dioic acid using $\left[1,16-{ }^{18} \mathrm{O}_{2}\right]$ PGE-MUM as an internal standard. This analysis showed that the urinary amounts of this new metabolite are at least as high as the amounts of PGE-MUM in urine from healthy children. This suggests that the synthesis of cyclooxygenase metabolites in children with ZS is comparable to that in healthy children. The consequences of these high amounts of abnormal cyclooxygenase metabolites in children with ZS is not clear. Most PG have been shown to be anticonvulsive (22); however, $\mathrm{PGF}_{2 \alpha}$ has been shown to induce grand mal-like seizures in humans $(23,24)$. In conclusion, the present paper shows that cyclooxygenase products are exclusively $\beta$-oxidized by the peroxisomal pathway in vivo in humans. We detected a novel metabolite of the E PG in urine of children with ZS. This metabolite is $\omega$-but not $\beta$-oxidized.

Acknowledgments. The authors are grateful to Drs. B. B. H. Schudgens and R. J. A. Wanders, Amsterdam, The Netherlands, for performing the specific biochemical diagnosis in the studied children. We thank M. Böhme for the excellent technical assistance.

\section{REFERENCES}

1. Oates JA, FitzGerald GA, Branch RA, Jackson EK, Knapp HR, Roberts LJ 1988 Clinical implications of prostaglandin and thromboxane $A_{2}$ formation (1). N Engl J Med 319:689-698

2. Oates JA, FitzGerald GA, Branch RA, Jackson EK, Knapp HR, Roberts LJ 1988 Clinical implications of prostaglandin and thromboxane $A_{2}$ formation (2). N Engl J Med 319:761-767

3. Bakhle YS 1983 Synthesis and catabolism of cyclo-oxygenase products. $\mathrm{Br}$ Med Bull 39:214-218 
4. Robinson C, Hardy CC, Holgate ST 1985 Pulmonary synthesis, release, and metabolism of prostaglandins. J Allergy Clin Immunol 76:265-271

5. Hamberg M 1968 Metabolism of prostaglandins in rat liver mitochondria. Eur J Biochem 6:135-146

6. Schepers L, Casteels M, Vamecq J, Parmentier G, Van Veldhoven PP, Mannaerts GP 1988 Beta-oxidation of the carboxyl side chain of prostaglandin $E_{2}$ in rat liver peroxisomes and mitochondria. J Biol Chem 263:2724-2731

7. Diczfalusy U, Alexson SE 1990 Identification of metabolites from peroxisomal beta-oxidation of prostaglandins. J Lipid Res 31:307-314

8. Lazarow P, Moser HW 1989 Disorders of peroxisome biogenesis. In: Scriver CR, Beaudet AL, Sly WS, Valle D (eds) The Metabolic Basis of Inherited Disease. McGraw-Hill, New York, pp 1479-1509

9. Wanders RJ, Schutgens RB, van den Bosch H, Tager JM, Kleijer WJ 1991 Prenatal diagnosis of inborn errors in peroxisomal beta-oxidation. Prenat Diagn 11:253-261

10. Jedlitschky G, Huber M, Völkl A, Müller M, Leier I, Müller J, Lehmann WD, Fahimi HD, Keppler D 1991 Peroxisomal degradation of leukotrienes by beta-oxidation from the omega-end. J Biol Chem 266:24763-24772

11. Mayatepek E, Lehmann WD, Fauler J, Tsikas D, Frölich JC, Schutgens RB, Wanders RJ, Keppler D 1993 Impaired degradation of leukotrienes in patients with peroxisome deficiency disorders. J Clin Invest 91:881-888

12. Serafin WE, Oates JA, Hubbard WC 1984 Metabolism of leukotriene $B_{4}$ in the monkey. Identification of the principal nonvolatile metabolite in the urine. Prostaglandins 27:899-911

13. Hamberg M, Samuelsson B 1971 On the metabolism of prostaglandins $E_{\text {, }}$ and $E_{2}$ in man. J Biol Chem 246:6713-6721

14. Rosenkranz B, Fischer C, Weimer KE, Frölich JC 1980 Metabolism of prostacyclin and 6-keto-prostaglandin $F_{1 \alpha}$ in man. J Biol Chem 255:10194 10198

15. Roberts LJ, Sweetman BJ, Oates JA 1981 Metabolism of thromboxane $B_{2}$ in man. Identification of twenty urinary metabolites. J Biol Chem 256:8384-8393
16. Uedelhoven WM, Meese CO, Weber PC 1989 Analysis of the major urinary thromboxane metabolites, 2,3-dinorthromboxane $\mathrm{B}_{2}$ and 11-dehydrothromboxane $B_{2}$, by gas chromatography-mass spectrometry and gas chromatography-tandem mass spectrometry. J Chromatogr 497:1-16

17. Frölich JC, Sawada M, Bochmann G, Oelz O 1986 Advances in the analysis of eicosanoids by ELISA and GC/MS/MS. In: Zor U, Naor Z, Kohen F (eds) Advances in Prostaglandin, Thromboxane and Leukotriene Research. Raven Press, New York, pp 363-371

18. Diczfalusy U, Kase BF, Alexson SE, Bjorkhem I 1991 Metabolism of prostaglandin $F_{2 \alpha}$ in Zellweger syndrome. Peroxisomal beta-oxidation is a major importance for in vivo degradation of prostaglandins in humans. J Clin Invest 88:978-984

19. Diczfalusy U, Vesterqvist O, Frode Kase B, Lund E, Alexson SEH 1993 Peroxisomal chain-shortening of thromboxane $\mathrm{B}_{2}$ : evidence for impaired degradation of thromboxane $\mathbf{B}_{2}$ in Zellweger syndrome. J Lipid Res 34:11071113

20. Catella F, Nowak J, FitzGerald GA 1986 Measurement of renal and non-renal eicosanoid synthesis. Am J Med 81:23-29

21. Frölich JC, Wilson TW, Sweetman BJ, Smigel M, Nies AS, Carr K, Watson JT, Oates JA 1975 Urinary prostaglandins; identification and origin. J Clin Invest 55:763-770

22. Hertting G, Seregi A 1989 Formation and function of eicosanoids in the central nervous system. Ann NY Acad Sci 559:84-99

23. Lyneham RC, Low PA, McLeod JG, Shearman RP, Smith ID, Korda AR 1973 Convulsions and electroencephalogram abnormalities after intraamniotic prostaglandin $\mathrm{F}_{2 \alpha}$. Lancet 2:1003-1005

24. Sederberg-Olsen J, Olsen CE 1983 Prostaglandin-oxytocin induction of midtrimester abortion complicated by grand mal-like seizures. Acta Obstet Gynecol Scand 62:79-81 